International Journal of Pure and Applied Mathematics

Volume 88 No. 4 2013, 557-567

ISSN: 1311-8080 (printed version); ISSN: 1314-3395 (on-line version)

url: http://www.ijpam.eu

doi: http://dx.doi.org/10.12732/ijpam.v88i4.10

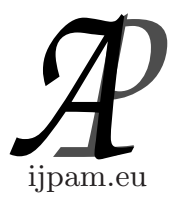

\title{
THE STABILITY PROPERTIES OF STRONG INVARIANT APPROXIMATION PROPERTY
}

\author{
Kankeyanathan Kannan \\ Department of Mathematics and Statistics \\ University of Jaffna \\ Jaffna, SRI LANKA
}

\begin{abstract}
Let $G$ be a countable exact discrete group. $G$ has the strong invariant approximation property(SIAP) if and only if

$$
C_{u}^{*}(G, S)^{G}=C_{\lambda}^{*}(G) \otimes S
$$

for any Hilbert space $\mathcal{H}$ and closed subspace $S \subseteq \mathcal{H}$. We shall use results of Haagerup and Kraus on the approximation property (AP) to investigate some permanence properties of the SIAP for discrete groups. This can be done most efficiently for exact groups. In this paper we describe that the stability properties of the SIAP property pass to semi direct products, and extensions for discrete exact groups.
\end{abstract}

AMS Subject Classification: 20F65, 18G60

Key Words: strong invariant approximation property, uniform Roe algebras, invariant approximation property

\section{Introduction}

The purpose of this paper is to provide an illustration of an interesting and nontrivial interaction between analytic and geometric properties of a group. We provide approximation property of operator algebras associated with discrete groups. There are various notions of finite dimensional approximation properties for $C^{*}-$ algebras and more generally operator algebras. Some of

Received: September 12, 2013

(c) 2013 Academic Publications, Ltd. url: www.acadpubl.eu 
these (approximation properties) notations will be defined in this paper, the reader is referred to $[2,9,8,4,12]$ for these a beautiful concept: Haagerup discovery that that the reduced $C^{*}-$ algebra $\mathbf{F}_{n}$ has the metric approximation property, Higson and Kasparov's resolution of the Baum-connes conjecture for the Haagerup groups. We studies analytic techniques from operator theory that encapsulate geometric properties of a group. On approximation properties of group $C^{*}$ - algebras is everywhere; it is powerful, important, backbone of countless breakthroughs.

Roe considered the discrete group of the reduced group $C^{*}$ - algebra of $C_{r}^{*}(G)$ is the fixed point algebra $\{A d \rho(t): t \in G\}$ acting on the uniform Roe algebra $C_{u}^{*}(G)[12]$. A discrete group $G$ has natural coarse structure which allows us to define the the uniform Roe algebra, $C_{u}^{*}(G)[12]$. We say that the uniform Roe algebra, $C_{u}^{*}(G)$, is the $C^{*}$ - algebra completion of the algebra of bounded operators on $\ell^{2}(X)$ which have finite propagation. The reduced $C^{*}-$ algebra $C_{r}^{*}(G)$ is naturally contained in $C_{u}^{*}(G)[12]$. According to Roe [12] $G$ has the invariant approximation property (IAP) if

$$
C_{\lambda}^{*}(G)=C_{u}^{*}(G)^{G}
$$

According to [15] $G$ has the strong invariant approximation property(SIAP) if and only if

$$
C_{u}^{*}(G, S)^{G}=C_{\lambda}^{*}(G) \otimes S
$$

for any Hilbert space $\mathcal{H}$ and closed subspace $S \subseteq \mathcal{H}$. We give a general exposition of invariant approximation property(IAP), which was initiated by Roe [12].

Our interest in these properties comes from a link to the strong invariant approximation property (SIAP) of Zacharias, which implies the IAP . We shall use results of Haagerup and Kraus [6] on the AP to investigate some permanence properties of the IAP and the SIAP for discrete groups. This can be done most efficiently for exact groups. In this paper we describe that the analytic properties of the SIAP property pass to semi direct products (see Proposition 3.8), and extensions for discrete exact groups (see Proposition 3.6).

\section{Preliminaries}

An important class of $C^{*}$ - algebras arises in the study of groups. Let $G$ be a discrete group, then the characteristic function $\delta_{g}(s)$ of $s \in G$ is defined as follows [4]: 


$$
\delta_{g}(s)= \begin{cases}1 & \text { if } g=s \\ 0 & \text { if } g \neq s\end{cases}
$$

If we assume that the $G$ is a discrete group then the functions $\delta_{g}$ form a basis for the Hilbert space $\ell^{2}(G)$ of square summable functions on $G$. The group ring $\mathbb{C}[G]$ consists of all finitely supported complex-valued functions on $G$, that is of all finite combinations $f=\sum_{s \in G} a_{s} s$ with complex coefficients. Denote $B\left(\ell^{2}(G)\right)$ the $C^{*}$ - algebra of all bounded linear operator on Hilbert space $\ell^{2}(G)$.

Definition 2.1. [4] The left regular representation

$$
\lambda: \mathbb{C}[G] \rightarrow B\left(\ell^{2}(G)\right)
$$

is defined by

$$
\lambda(s) \delta_{t}(r)=\delta_{t}\left(s^{-1} r\right)=\delta_{s t}(r) \text { for } s, r \in G . .
$$

The right regular representation is given by

$$
\rho(s) \delta_{t}(r)=\delta_{t}(r s)=\delta_{t s^{-1}}(r) \text { for } s, r \in G .
$$

The reduced $C^{*}$ - algebra $C_{\lambda}^{*}(G)$ of a group $G$ (which we shall assume to be discrete) arises from the study of the left regular representation $\lambda$ of the group ring $\mathbb{C}[G]$ on the Hilbert space of square-summable functions on the group.

Definition 2.2. [4] The reduced group $C^{*}$ - algebra $G$, denoted by $C_{r}^{*}(G)$ is the completion of $\mathbb{C}[G]$ in the norm given, for $c \in \mathbb{C}[G]$, by

$$
\|c\|_{\lambda}=\|\lambda(c)\|
$$

Equivalently, it is the closure of $\mathbb{C}[G]$ is identified with its image under the left regular representation. i.e.

$$
C_{\lambda}^{*}(G):=\overline{\lambda(\mathbb{C}[G])}
$$

The reduced $C^{*}$ - algebra $C_{\rho}^{*}(G)$ of a group $G$ (which we shall assume to be discrete) arises from the study of the right regular representation $\rho$ of the group ring $\mathbb{C}[G]$ on the Hilbert space of square-summable functions on the group.

Definition 2.3. The reduced group $C^{*}-$ algebra $G$, denoted by $C_{\rho}^{*}(G)$ is the completion of $\mathbb{C}[G]$ in the norm given, for $c \in \mathbb{C}[G]$, by

$$
\|c\|_{\rho}=\|\rho(c)\| .
$$


Equivalently, it is the closure of $\mathbb{C}[G]$ is identified with its image under the right regular representation.

$$
C_{\rho}^{*}(G):=\overline{\rho(\mathbb{C}[G])}
$$

In this section we shall establish the basic definitions and notations for the category of coarse metric spaces. Coarse geometry is the study of the large scale properties of spaces. The notion of large scale is quantified by means of a coarse structure.

Example 2.4. [12] Let $G$ be a finitely generated group. Then the bounded coarse structure associated to any word metric on $G$ is generated by the diagonals

$$
\Delta_{g}=\{(h, h g): h \in G\}
$$

as $g$ runs over $G$.

Let $X$ be a discrete metric space.

Definition 2.5. [12] We say that discrete metric space $X$ has bounded geometry, if for all $R$ there exists $N$ in $\mathbb{N}$ such that for all $x \in X,\left|B_{R}(x)\right|<N$, where $B(x, R)=\{x \in X: d(y, x) \leq R\}$.

Definition 2.6. [12] A kernel $\phi: X \times X \longrightarrow \mathbb{C}$

- is bounded, if there exists $M>0$ such that $|\phi(s, t)|<M$ for all $s, t \in$ $X$.

- has finite propagation, if there exists $R>0$ such that $\phi(s, t)=0$ if $d(s, t)>R$.

Let $B(X)$ be a set of bounded finite propagation kernels on $X \times X$. Each such $\phi$ defines a bounded operator on $\ell^{2}(X)$ via the usual formula for matrix multiplication

$$
\phi * \zeta(s)=\sum_{r \in G} \phi(s, r) \zeta(r), \text { for } \zeta \in \ell^{2}(X) .
$$

Next, we show the operator associated with a bounded kernel is bounded.

Lemma 2.7. [8] Let $X$ be bounded geometry metric space. An operator associated with a bounded finite propagation kernel is bounded.

We shall denote the finite propagation kernels on $X$ by $A^{\infty}(X)$.

Definition 2.8. The uniform Roe algebra of a metric space $X$ is the closure of $A^{\infty}(X)$ in the algebra $B\left(\ell^{2}(X)\right)$ of bounded operators on $X$. 
If a discrete group $G$ is equipped with its bounded coarse structure introduced in Example 2.4 then one can associated with it uniform Roe algebra $C_{u}^{*}(G)$ by repeating the above.

A discrete group $G$ has a natural coarse structure which allows us to define the uniform Roe algebra $C_{u}^{*}(G)$. A group $G$ can be equipped with either the left or right-invariant of the metric. A choice of one of the determines whether $C_{\lambda}^{*}(G)$ or $C_{\rho}^{*}(G)$ is a sublagebra of the uniform Roe algebra $C_{u}^{*}(G)$ of $G$.

We now define the invariant approximation property (IAP)

Definition 2.9. [12] We say that $G$ has the invariant approximation property $(I A P)$, if

$$
C_{\lambda}^{*}(G)=C_{u}^{*}(G)^{G}
$$

Roe [12] is proved in that amenable groups and finitely generated free groups have the IAP. Haagerup and Kraus [7] defined and studied the AP properties (AP) for a group. We begin with a some definition of Haagerup and Kraus [7].

Definition 2.10. [1] A $C^{*}$ - algebra $A$ is nuclear if and only if it has the following completely positive approximation property (CPAP): The identity map on $A$ can be approximated in the point norm topology by finite rank completely positive contractions. This means that there exist nets of operators $T_{\alpha}: A \longrightarrow M_{n_{\alpha}}(\mathbb{C})$ and $S_{\alpha}: M_{n_{\alpha}}(\mathbb{C}) \longrightarrow A$ such that for all $a \in A$

$$
\lim _{\alpha}\left\|S_{\alpha} T_{\alpha}(a)-a\right\|=0 .
$$

A $C^{*}$ - algebra $A$ has the metric approximation property (MAP) of Grothendieck if and only if the identity map on $A$ can be approximated in the point-norm topology by a net of finite rank contractions.

Comparing the definitions we see that CPAP implies MAP (see for example [1]). Lance [11] has shown that $\Gamma$ is amenable if and only if its reduced $C^{*}-$ algebra $A$ has the CPAP which is equivalent to $C_{r}^{*}(\Gamma)$ being nuclear. Completely positive maps are in particular completely bounded, which suggest the following weakening of the CPAP.

Definition 2.11. [1] A $C^{*}$-algebra $A$ is said to have the completely bounded approximation property (CBAP) if there is a positive number $C$ such that the identity map on $A$ can be approximated in the point norm topology by a net $\left\{\phi_{\alpha}\right\}$ of finite rank completely bounded maps whose completely bounded norms are bounded by $C$. This means that there exists a net of finite-rank maps $\left\{\phi_{\alpha}\right\}: A \longrightarrow A$ such that $\left\|\phi_{\alpha}\right\|_{c b} \leq C$ for some constant $C$ and $\phi_{\alpha} \longrightarrow i d_{A}$ in the point-norm topology on $A$ (i.e. $\left\|\phi_{\alpha}(x)-x\right\| \longrightarrow 0$ for all $x \in A$ ). 
We have the following definition of weak amenability.

Definition 2.12. [1] An approximate identity on $G$ is a sequence $\left(\phi_{n}\right)$ of finitely supported functions such that $\phi_{n}$ uniformly converge to constant function 1 . We say that discrete $G$ is weakly amenable if there is an approximate identity $\left(\phi_{n}\right)$ such that

$$
C:=\sup \left\|M_{\phi_{\mathrm{n}}}\right\|_{c b}<\infty \text {. }
$$

We have the following important result by Haagerup [6].

Theorem 2.13. Let $G$ be a discrete group. The following are equivalent:

1. $G$ is weakly amenable,

2. $C_{r}^{*}(G)$ has the $C B A P$.

Definition 2.14. [2] We say that discrete group $G$ is amenable, if and only if there is an approximate identity consisting of positive definite functions.

Lemma 2.15. [2] An amenable discrete group is weakly amenable.

We have the following definition of operator approximation property (OAP) and approximation property (AP).

Definition 2.16. [7] We say that $C^{*}$ - algebra, $A$ has the operator approximation property (OAP), if there exists a net of finite - rank maps $T_{\alpha}: A \longrightarrow A$ such that $T_{\alpha} \longrightarrow i d_{A}$ in the stable point-norm topology. This means that there exists a net of finite rank linear maps

$$
T_{\alpha}: A \longrightarrow A
$$

such that for all $x \in \mathcal{K}(\mathcal{H}) \otimes_{\min } A$,

$$
\left\|I d \otimes T_{\alpha}(x)-x\right\| \longrightarrow 0 .
$$

The Fourier algebra

$$
A(G):=\left\{f: f(t)=\langle\lambda(t) \xi \mid \eta\rangle \text { for some } \xi, \eta \in \ell_{2}(G)\right\}
$$

is the space of all coefficient function of the left regular representation $\lambda$. Given $f \in A(G)$, its norm is given by

$$
\|f\|=\inf \{\|\xi\|\|\eta\|: \quad f(t)=\langle\lambda(t) \xi \mid \eta\rangle\} .
$$

With this norm, $A(G)$ is a Banach algebra with the pointwise multiplication $[7]$. 
Definition 2.17. [7] A complex-valued function $\phi$ on $G$ is a multiplier for $A(G)$, if the linear map

$$
M_{\phi}(f)=\phi f
$$

sends $A(\Gamma)$ to $A(\Gamma)$. If the map $M_{\phi}$ is completely bounded on $A(G)$, we call $\phi$ a completely bounded multiplier of $A(G)$. The set of multipliers of $A(G)$ is denoted by $M_{0} A(G)$. If $\phi \in A(G)$ then $\phi$ is a bounded continuous function and $M_{\phi}$ is a bounded operator on the space $A(G)$.

Definition 2.18. [7] The discrete group $G$ has the approximation property (AP) if there is a net $\left\{\phi_{\alpha}\right\}$ in $A(G)$ such that $M_{\phi_{\alpha}} \longrightarrow i d_{A(G)}$ in the stable point-norm topology on $A(G)$.

The following implication for discrete groups [15]:

Theorem 2.19.

$$
C B A P \Rightarrow A P \Rightarrow I A P .
$$

We then use this to show the following groups have invariant approximation property [10]:

- Amenable groups

- Hyperbolic groups

- $C A T(0)$-cubical groups $[2]$

- $S L_{2}\left(\mathbb{Q}_{p}\right)[3]$.

In this section we will give definition of the strong invariant approximation property. Let $S \subseteq B(\mathcal{H})$ be a closed subspace.

Next, we define the set of fixed points of $C_{u}^{*}(G, S)^{G}$ :

Definition 2.20. We define

$$
C_{u}^{*}(G, S)^{G}=\left\{T \in C_{u}^{*}(G, S) ; \operatorname{Ad}\left(\rho_{t} \otimes i d\right) T=T \text { for all } t \in G\right\} .
$$

We now define Joachim Zacharias's IAP with coefficients (SIAP):

Definition 2.21. [15] We say that a discrete group $G$ has the strong invariant approximation property (SIAP) if for any closed subspace $S$ of the compact operators $\mathcal{K}\left(\right.$ on $\left.\ell^{2}(\mathbb{N})\right)$. We have an isomorphism

$$
C_{u}^{*}(G, S)^{G}=C_{\lambda}^{*}(G) \otimes S \text { holds. }
$$




\section{The Stability Properties of Strong IAP}

In this section, we show some of the stability properties of the strong invariant approximation property for discrete exact groups.

Theorem 3.1. [15]For a discrete exact group $G$ the following are equivalent.

1. $G$ has the AP.

2. $C_{r}^{*}(G)$ has the $O A P$.

3. G has SIAP (Zacharias's IAP with coefficients)

Theorem 3.2. For a discrete exact group $G$. $G$ has the SIAP if for any closed subspace $S \subseteq B(\mathcal{H})$ the equality

$$
C_{u}^{*}(G, S)^{G}=C_{\lambda}^{*}(G) \otimes S \text { holds. }
$$

Theorem 3.3. [15] SIAP implies IAP for discrete exact groups.

We note also the following results.

Theorem 3.4. [2] Let $G$ be a discrete group. Let

$$
1 \longrightarrow H \longrightarrow G \stackrel{\pi}{\longrightarrow} G / H \longrightarrow 1 \text {. }
$$

If $H$ is a normal subgroup in $G$ with $H$ and $G / H$ are exact, then $G$ is exact.

We have the following important result of Haagerup and Kraus [7].

Theorem 3.5. Let $G$ be locally compact group, and suppose that $H$ is a closed normal subgroup of $G$. If $H$ and $G / H$ have the $A P$, then $G$ has the $A P$.

In the following Proposition, we show that the strong invariant approximation property (SIAP) passes to extensions for discrete exact groups.

Proposition 3.6. Let $G$ be a discrete group. Let

$$
1 \longrightarrow H \longrightarrow G \stackrel{\pi}{\longrightarrow} G / H \longrightarrow 1 \text {. }
$$

Let us assume that $H$ is a normal subgroup in $G$, and that $H$ and $G / H$ are exact groups. If $H$ and $G / H$ have the SIAP, then $G$ has SIAP.

Proof. Let $G$ be a discrete group and suppose that $H$ is a normal subgroup of $G$. By Theorem 3.1, if $H$ has the SIAP then $H$ has AP. If $G / H$ has the SIAP then $G / H$ has the AP. By Theorem 3.5, if $H$ and $G / H$ have the AP, 
then $G$ has the AP. By Theorem 3.4, if $H$ and $G / H$ are exact groups, then so is $G$. Thus $G$ is a discrete exact group with AP. By Theorem 3.1, $G$ has SIAP.

In this section, we first show that the semidirect product of two discrete exact groups with the SIAP has the SIAP. We have the following important result in $[7]$.

Proposition 3.7. The semidirect product of two discrete groups with the $A P$ has the AP.

From this we can deduce the following.

Proposition 3.8. The semidirect product of two discrete exact groups with the SIAP has the SIAP.

Proof. For an exact group $G, G$ has AP if and only if $G$ has SIAP. The semidirect product of two discrete groups with the AP has the AP [7].

The semidirect product of two discrete exact groups is an exact group [14].

The semidirect product of two discrete groups with the AP has the AP (by Proposition 3.5). Therefore the semidirect product of two discrete exact groups with the SIAP has the SIAP.

We propose a simple method to establish that the following group have approximation property (AP).

Example 3.9. [7] We have the following short exact sequence of groups

$$
1 \longrightarrow \mathbb{Z}^{2} \longrightarrow \mathbb{Z}^{2} \rtimes S L(2, \mathbb{Z}) \longrightarrow S L(2, \mathbb{Z}) \longrightarrow 1 .
$$

Indeed, $\mathbb{Z}^{2}$ and $S L(2, \mathbb{Z})$ are weakly amenable groups [7]. But $\mathbb{Z}^{2} \rtimes_{\rho} S L(2, \mathbb{Z})($ where $\rho$ is the standard action of $S L(2, \mathbb{Z})$ on $\left.\mathbb{Z}^{2}\right)$ can be written as an amalgamated free product

$$
\mathbb{Z}^{2} \rtimes S L(2, \mathbb{Z})=\mathbb{Z}^{2} \rtimes\left(\mathbb{Z}_{4} *_{\mathbb{Z}_{2}} \mathbb{Z}_{6}\right)=\mathbb{Z}^{2} \rtimes \mathbb{Z}_{4} *_{\mathbb{Z}^{2} \rtimes \mathbb{Z}_{2}} \mathbb{Z}^{2} \rtimes \mathbb{Z}_{6},
$$

whose factors are amenable and also $\Lambda\left(\mathbb{Z}^{2} \rtimes S L(2, \mathbb{Z})\right)=\infty[7]$. So, the class of groups $C^{*}$-algebra that are weakly amenable is not closed under taking arbitrary amalgamated free product. The semidirect product of two discrete groups with the AP has the AP [7]. Thus, $\mathbb{Z}^{2} \rtimes S L(2, \mathbb{Z})$ has AP [7]. By using Proposition 3.7. Therefore $\mathbb{Z}^{2} \rtimes S L(2, \mathbb{Z})$ has AP In other words: $\mathbb{Z}^{2}$ and $S L(2, \mathbb{Z})$ have CBAP. Then by using $2.19, \mathbb{Z}^{2}$ and $S L(2, \mathbb{Z})$ have AP. 


\section{References}

[1] J. Brodzki and G. A. Niblo, Approximation properties for discrete groups, $C^{*}$-algebras and elliptic theory, Trends Math, Basel, (2006), 23-35.

[2] P. Brown and N. G. Ozawa, $C^{*}$ and Finite Dimensional Approximations, Graduate Studies in Mathematics, American Mathematical Soc., 88 (2008).

[3] M. Bożejko and M. A.Picardello, Weakly amenable groups and amalgamated products. Proc, Amer. Math. Soc. 117 (1993), 1039-1046.

[4] K. R. Davidson, $C^{*}$ - algebra by Example, Field Institute Mono-graphs, American Mathematical Soc, Providence, RI, 6 (1986).

[5] E. Guentner, N. Higson and S. Weinberger, The Novikov conjecture for linear groups, Publ. Math. Inst. Hautes Études Sci, 101 (2005), 243-268.

[6] U. Haagerup, An example of a non nuclear $C^{*}$-algebra, which has the metric approximation property, Inventiones Mathematicae, 50 (1978), nu 3, 279-293.

[7] U. Haagerup and J. Kraus, Approximation Properties for Group $C^{*}$ Algebras and Group Von Neumann Algebras, Inventiones Mathematicae, 344 (1994), nu 2, 667-699.

[8] K. Kannan, On approximation properties of group $\mathrm{C}^{*}$ - algebras, University of Southampton, School of Mathematics, Doctoral Thesis, (2011), nu 2, 116 pp.

[9] K. Kannan, Strong Invariant Approximation Property for Discrete Groups, International Journal of Pure and Applied Mathematics, 85 (2013), 10751086.

[10] K. Kannan, A Brief Survey of Approximation Properties, Mathematical Sciences International Research Journal, 6 (2013), 1075-1086.

[11] E. C. Lance, On nuclear $C^{*}$ - algebras, Journal of Functional Analysis, 12(1973), 157-176.

[12] J. Roe, Lectures on coarse geometry, American Mathematical Society, 31 (2003). 
[13] A. Valette, Introduction to the Baum-Connes conjecture, Lectures in Mathematics ETH Zürich, (2002).

[14] G. Yu, The coarse Baum-Connes conjecture for spaces which admit a uniform embedding into Hilbert spaceIntroduction to the Baum-Connes conjecture, Invent. Math., 139 (2000), 201-240.

[15] J. Zacharias, On the Invariant Translation approximation property for Discrete Groups, Proc. Amer. Math. Soc., 134 (2006), nu 7, 1909-1916. 
